\title{
Review on Light Fidelity (Li-Fi) - An Advancement of Wireless Network
}

\author{
Ramandeep Kaur ${ }^{\mathrm{a}}$, Himanshu Walia ${ }^{\mathrm{b}}$ \\ ${ }^{a}$ Research Scholar, Kapurthala,144601, India \\ ${ }^{b}$ Research Scholar,Bathinda, 1516001, India
}

\begin{abstract}
Nowadays, Li-Fi is becoming very popular and catching the interest of a number of users with its advanced technology-based features. It brings a new resolution in the wireless communication and people are showing so much curiosity to know about it. $\mathrm{Li}-\mathrm{Fi}$ is an optical wireless communication-based technology which transmits data over the network through a light source like LED's instead of radio frequency signals (RF) with very high data rate. The aim of this paper is to give full- fledged knowledge about Li-Fi \& its applications. This paper also attempts to clarify the basic difference between wireless technologies and their evolution $\&$ how this new technology is leading all over the world with its features like less power consumption, less requirement of maintenance, \& bi-directional in nature. Li-Fi eliminates the effect of harmful radiations that occur due radio signals as in $\mathrm{Wi}-\mathrm{Fi}$ and has less latency rate. This paper gives an overview and the general idea about working on Li-Fi technology and how it will help in the development of bright future as it is beyond the expectations of end users.
\end{abstract}

Index Terms: Light Fidelity, Data Security, Light Emitted Diodes, Photo Detector, Wireless Network, Light Communication.

(C) 2017 Published by MECS Publisher. Selection and/or peer review under responsibility of the Research Association of Modern Education and Computer Science

\section{Introduction}

Li-Fi (Light Fidelity) is a new era and future of wireless communication with high-speed and bi-directional access. It is an advanced and optical network based technology that relates to Wi-Fi (Wireless Fidelity) [1]. Instead of using radio frequency signals, Li-Fi sends data and provides network communication using visible light signals. It's speed is 100 times more than Wi-Fi with wider bandwidth i.e. approximate 224 gigabits per second speed. It is based on optical wireless communication protocol (OWC) in which Light emitting Diodes (LED's) are used to obtain light signals as a medium to transmit data over network wirelessly. The idea of

* Corresponding author.

E-mail address: raman177rk@gmail.com;himanshuwalia792@gmail.com 
wireless communication by light was firstly demonstrated by Dr. Herald Haas (Germen Physicist) at the University of Edinburgh (UK) in TED Google Talk on VLC to overcome the problem of the limited availability of radio signals based on electromagnetic spectrum and to promote the idea of optical wireless based communication systems [9]. Li- Fi supports to the same IEEE standard as Wi-Fi do i.e. IEEE 802.11 standards. It is also known as visible light communication system (VLC) as it uses the visible light and ultraviolet rays for transmission whose frequency lies in between $400 \mathrm{THz}$ to $800 \mathrm{~Hz}$ in electromagnetic spectrum as shown in Fig 1.

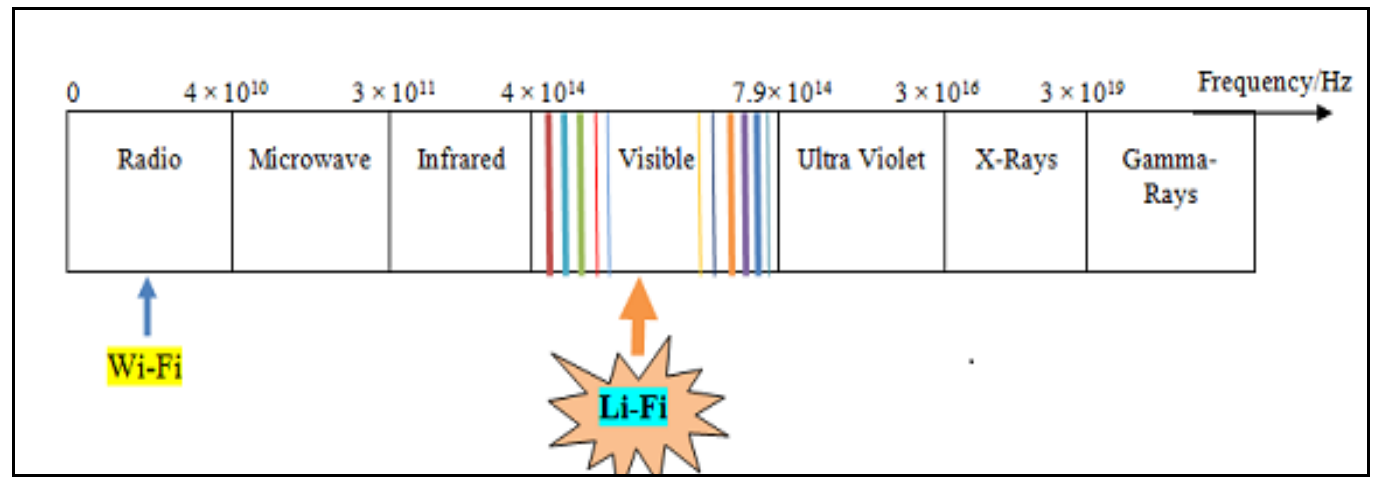

Fig.1. Electromagnetic Spectrum of Li-Fi

Its main advantage is to access the internet at very high speed through visible light signals like LED lights equipment. Now instead of accessing your internet through Wi-Fi signals, we will be able to access your internet through light source such as your room LED bulbs, Torchlight $\&$ flashlight of your phones, car lights etc with very high data rates and speed and mostly useful for those regions where optical fibre cables are not easy to install like hill station areas.

\section{Evolution of Wireless Technologies}

From the past years ago, a number of wireless technologies invented to enhance the technical world. The radio frequency based wireless communication brings a great revolution in network field and becoming popular day by day as regular activities of users are getting very frequent with wireless communication. It is helping them to lift their lifestyle in very short time and very rapidly. There are so many wireless technologies are available that come in existence. Before going through their personal characteristics and benefits, we must know how they come into existence with new benefits.

\section{A. Bluetooth}

Bluetooth is a short distance wireless communication technology used for sharing data over small local area network and come in existence in 1994 and demonstrated by Swedish company's group of members in Ericson. It replaces the cables or wires to connect the devices with the system in a personal local area network. It operates at $2.45 \mathrm{GHz}$ frequency with range 10 meters to 32 feet approximately. It support to IEEE standard IEEE 802.15.1 as shown in Fig 2 and uses frequency hopping spread spectrum (FHSS) based radio technology. The advanced version of Bluetooth is launched with enhanced RF Technology and data rate up to 24 Mbps such as Bluetooth $\mathrm{v} 1.0$ to Bluetooth $\mathrm{v}$ 4.1. It has the limitation of usability only at short distance communication [8]. 


\section{B. Cellular Networks}

It is a mobile based wireless technology which is used to provide mobile and very far distance communication. It is radio wave based wireless network and covers 9 to 21 miles geographical area. It was invented in 1901, after the invention of radio transmission by Guglielmo Marconi who used the concept of electromagnetic radiation (Clark Markwell, in 1985). 1 G, 2G, 3G, 4G and 4G LTE (Long Term Evolution) are the cellular networks which are used by cellular organizations to provide high-speed signals communication to their customers with high distance.

\section{Zigbee}

Zigbee is also a wireless technology that is used by industries, medical fields etc with low cost and low power requirements. It was ratified in 2004 by Zigbee alliance and launched for the use of the public in December 2005. Bluetooth was invented for to exceed and fast the data rate transfer for mobile phones and Zigbee was invented for slower the data rate i.e. 250kbps. It supports to IEEE 802.15.4 standard with maximum range coverage is 20 meters. It has the limitation of low data rate and works only indoor.

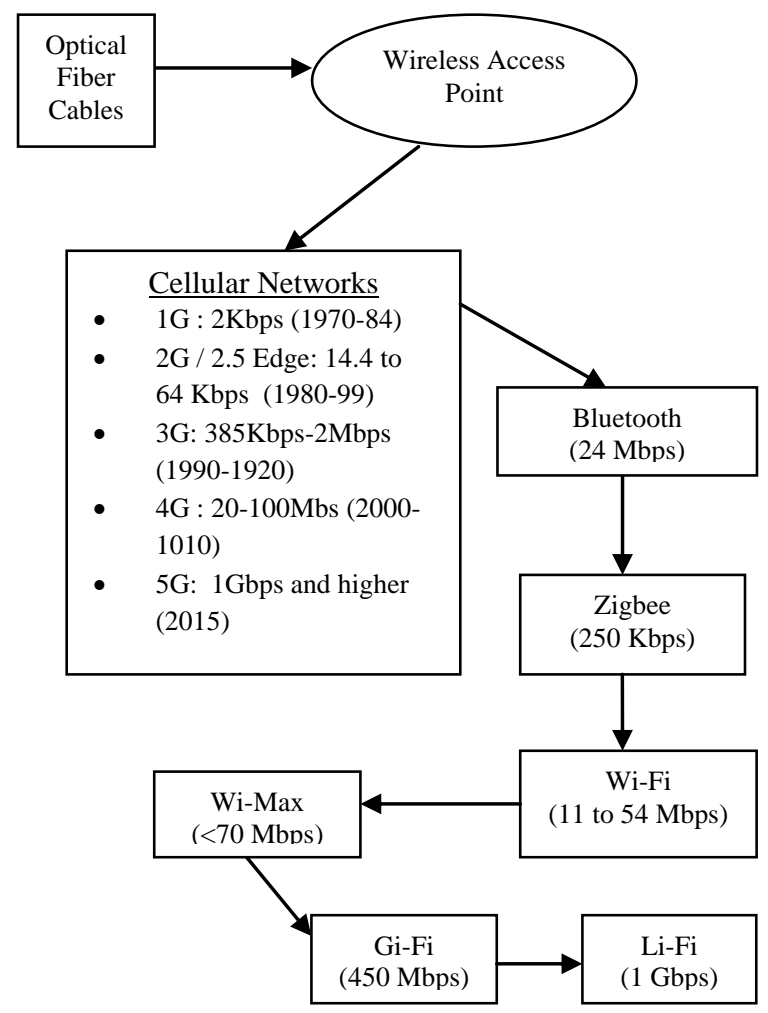

Fig.2. Evolution of Wireless Technology

D. Wi-Fi 
Wi-Fi is very popular radio wave based wireless communication network technology all over the world that is used to transfer data at the very high speed and for fast internet access and wirelessly. It was invented by NCR Corporation and AT\&T in Netherland (1991). It stands for Wireless Fidelity and supports to IEEE 802.11x standard with $2.5 \mathrm{Ghz}$ or $5 \mathrm{GHZ}$ frequency, 11 to $54 \mathrm{Mbps}(<=200 \mathrm{mbps})$ data rate and range $120-150$ feet. It enhanced version are IEEE 802.11a (operate using Orthogonal Frequency Division Multiplexing mechanisms: OFDM), IEEE 802.b, IEEE 802.11g and IEEE 802.11n [11].

\section{E. $\quad \mathrm{Gi}-\mathrm{Fi}$}

It is also known as Gigabit Wi-Fi or 5G Wi-Fi and supports to IEEE 802.11ac standard. It was demonstrated in 2004 by IEEE with advantages of less interference and very faster internet access and data transfer with data rate i.e. $450 \mathrm{Mbps}$ (near about Gigabits) and give very high throughput value to its end users. It is an advanced and extended version of Wi-Fi.

\section{F. Wi-Max}

Wi-Max stands for Worldwide Interoperability for Microwave Access and supports to IEEE 802.16 standard. It was come in existence in April 2001 and developed by group members of IEEE institute. It is used to provide broadband access to internet users with high-speed data transfer rate i.e. $<70 \mathrm{Mbps}$ and maximum distance coverage up to $3 \mathrm{Km}$. It provides 10 to $66 \mathrm{GHZ}$ frequency and its advance version are IEEE 802.16a, IEEE 802.16d-2004 \& 802.16e-2005. Wi-Max provides high quality and performance to VOIP (Voice over IP) related applications for video conferencing etc by using OFDM and MIMO (Multiple Input and Multiple Output) mechanisms [8].

\section{How Li-Fi Works?}

The working of Li-Fi technology is very simple as Wi-Fi. There are two basic components are used: LED Light Source \& Light sensor (Photodetector). The light source is work at one end and detector on another end. When LED bulb is on it starts glowing and the light sensor senses the light from light source and receives signals in the form of binary signals i.e. 1 or 0 . When some data is transmitted over the network from the internet to the user device, it transmits over the network and flashing of LED bulb is an indication of the message and then photodetector sense light and receive the message and forward to its destination end as shown in Fig 3. The main components of Li-Fi are:

- $\quad$ LED Lamp

- $\quad$ Photo Detector (Light Sensor)

- $\quad$ End-user Devices (Laptop, Computer, Mobile etc).

- Optical Wireless Communication protocols (OWC)

- Radio Frequency Signal's Amplifier

- $\quad$ Line of Sight Mechanism

More the brighter LED bulb, More frequently data will transmit light signals over the network and highly reliable.

\section{Li-Fi vs. Wi-Fi}

Li-Fi and Wi-Fi both are wireless communication networks, but are different from each other on the basis of the electromagnetic spectrum. Wi-Fi uses radio signals and transmits data using Wi-Fi Router (Access Point) 
and Li-Fi uses visual light signals for communication and uses light drivers like LED bulbs for transmission of data. Wi-Fi is mostly used for internet access like Wi-Fi hotspot etc but $\mathrm{Li}-\mathrm{Fi}$ is used in that areas where implementation of fiber optics is not possible such as airlines, submarines, undersea water \& hospitals etc for internet access and communication [2].

In Wi-Fi, Radio frequency signals (RF) can easily penetrate through walls of the building and can't be blocked so they have less security and to provide it security some security standards like WEP (Wireless encryption protocol), WAP2 etc are used to protect the data. In Li-Fi, light signals are blocked by the building walls so provide high security than $\mathrm{Wi}-\mathrm{Fi}$ and no need to use extra security standards for protecting it from unauthorized users. Wi-Fi has data speed up to $150 \mathrm{Mbps}$ and Li-Fi has data speed about 1 Gbps with 1000 time wider bandwidth.

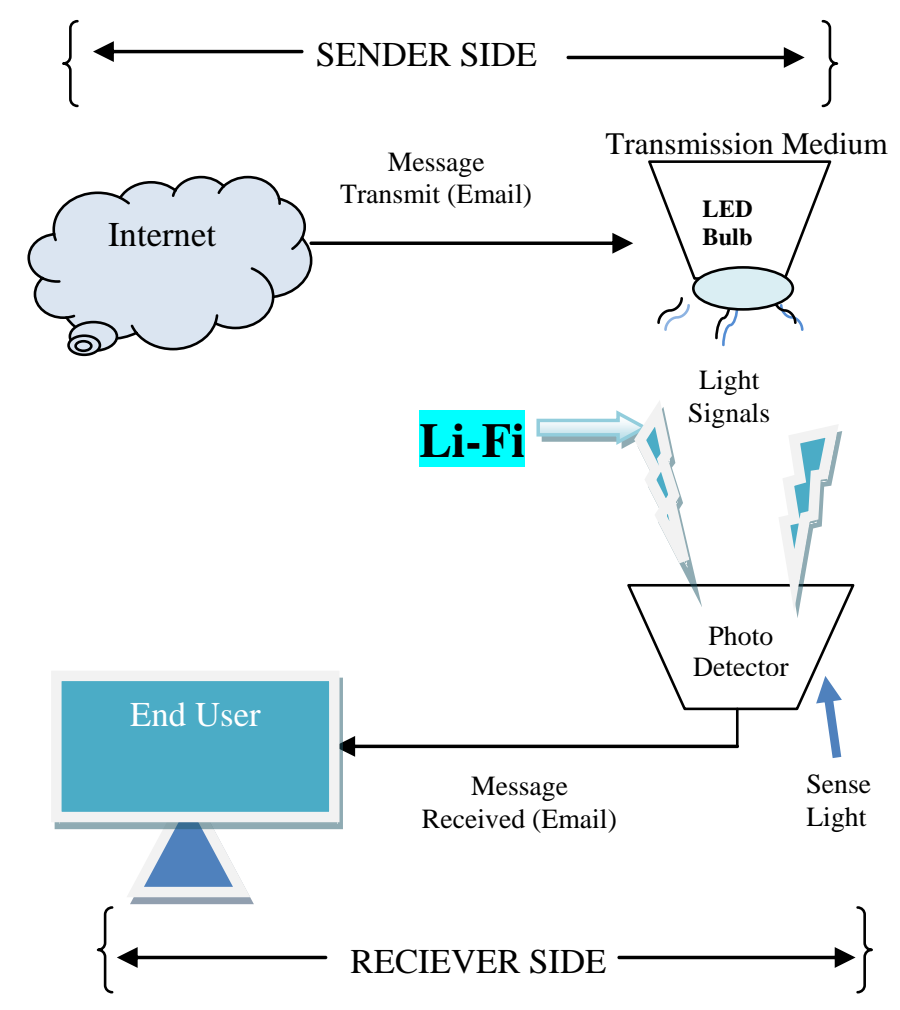

Fig.3. Working of Li- Fi

\section{Related Work on Li-Fi}

$\mathrm{Li}-\mathrm{Fi}$ is attracting the great interest of a number of users as compared to Wi-Fi. It is helping to overcome the problems of Wi-Fi system such as Low data rate and bandwidth, interference between multiple accesses points (AP's) and a number of limited channels for transmission using radio signals. Li-Fi playing an important role to overcome the shortage of radio signals bandwidth by using light source. Its implementation is getting the start and making a great revolution in wireless network filed. Numerous researchers and scientists are doing their great work done to solve the critical issue of Li-Fi. Some enhancement technologies and issues are discussed in various research papers which are explained as below with the great contribution of researchers: 
I. Ravi Parkash and Prachi Agarwal [4], have proposed a new technique to creating the Li-Fi network using Array LED's and TED based method.

- Problem Formulated: They formulate the problem of low data rate and speed to transmit data using one by one LED sources.

- Solution: To avoid this problem, the basic implementation of Li-Fi is enhanced by using Array-based LED's to transmit data parallel where each LED source transmit different data stream and help to achieve high data rates i.e. up to $10 \mathrm{Gbps}$. Also, the R, G, B LED's are used as a mixture to change the frequency of signals to transmit different data stream on different channels and increase data transmission speed thousand times more than standard data stream rate of bit i.e. 10,000 to 20,000 bps.

II. B.Narmada, P.Srinivasulu [6] has proposed highly efficient techniques for Li-Fi \& Wi-Fi using FINCH protocol. FINCH is a mobile IP-based protocol and reduce the latency rate and very suitable for real-time mobile applications to launch Li-Fi technology. The resultant value of this paper concludes that the novel methodology for LI-Fi with color shift keying (CSK) modulation can provide $12 \mathrm{Mbps}$ to $96 \mathrm{Mbps}$ data rate and also it has no need of IP encapsulation so provide high security.

III. Mushammad Usama, Kamran Saeed et. [17] has proposed a new method for Li-Fi using new technology i.e. Mobility in IP-based Communication Approach.

- Problem Formulated: They formulated a problem in Li-Fi technology i.e. loss of connection due to obstacle occur between sender and receiver as light signals can't penetrate through the building walls due to which loss of connectivity in mobile users takes place.

- Solution: The authors solved this problem by using the concept of redundant connectivity of multiple LED's as they will provide continuous connectivity by connecting one Li-Fi access point to another by using mobile in IP-based communication approach in which if home agent (HA) find any obstacle in connection then he will automatically connected to foreign agent (FA) to retrieve connection in case of long distances. They also focused on another scenario in which multiple LED's lamps can be installed for emergency use. It due to any obstacle one LED shut down than other will operate it; otherwise remain in sleep mode until the problem is not found and avoid the nomadic access problem i.e. use of Li-Fi within a specifically defined range.

\section{Security Concern of Li-Fi}

Li-Fi network is highly secured than other wireless networks like Wi-Fi, Bluetooth, and Wi-Max etc. Its security features are described as follow:

- $\quad$ Li-Fi is not public Li-Fi. It only works in indoor instead of outdoor so it can implement in regions or build a nuclear plant. Military etc. where high security is required as it drops the chances of system hacking [26].

- Its data transmission occur at very high speed i.e in nanoseconds which can't be detectable by the human visual system so human eye can't observe how data stream transmission is happening. They can only observe light and it will secure the system from cyber criminal's attacks and resist data manipulation over the network.

- It has no need of extra encryption standards like Wi-Fi because it has itself inbuilt security feature i.e. resistance against outdoor unauthorized usability. 


\section{Recent Researches on Li-Fi Technology}

Li-Fi is becoming very commercial technology and giving a bright future to end users with its speed enhancement facility over the world.

- Heinrich Hertz Institute (Berlin, Germany) - According to research, The group member of organization has implemented the concept of Haas and are able to transfer data with high rate speed up to $150 \mathrm{Mbps}$ using white LED Bulbs and they conclude that in future, Li-Fi will be able to transmit data rate i.e. 1 Gbps which will help to download any movie (HD) from internet just in 30 seconds [5].

- University of Scotland: They are also adopting the Li-Fi technology to achieve high-speed transmission of data on their campus for research scholars.

- In Los Vegas (2012:USA), a group of members uses Casio smartphone for transmitting the data using light and achieve its transmission range up to 10 meters.

- On research, it also has been researched that an organization named as Li-Fi Consortium is helping to promote $\mathrm{Li}$-Fi in various organizations, institutes etc.

Table 1. Evolution \& Comparison of Wireless Network

\begin{tabular}{|c|c|c|c|}
\hline Parameters & Bluetooth & $W i-F i$ & $L i-F i$ \\
\hline Evolution & $\begin{array}{l}1994 \text { by Group of } \\
\text { engineers at Ericsson, (a } \\
\text { Swedish company) }\end{array}$ & $\begin{array}{c}1991 \text { by NCR and AT\&T } \\
\text { Corporation }\end{array}$ & 2011 by Dr. Herald Haas \\
\hline Meaning & $\begin{array}{c}\text { Wireless small distance } \\
\text { network }\end{array}$ & Wireless Fidelity & Light Fidelity \\
\hline Medium & Radio signals & Radio Signals & Visible Light Signals \\
\hline Data Speed & 3 to $25 \mathrm{Mbps}$ & $250 \mathrm{Mbps}$ & $124 \mathrm{Gbps}$ \\
\hline Components & Bluetooth device & Wi-Fi Router & $\begin{array}{l}\text { LED Lamps \& Light } \\
\text { Sensor }\end{array}$ \\
\hline Bandwidth & $2.45 \mathrm{GHz}$ & $\begin{array}{c}100 \text { times wider than } \\
\text { Bluetooth }\end{array}$ & $\begin{array}{l}1000 \text { time wider than Wi- } \\
\text { Fi }\end{array}$ \\
\hline Cost & Very Less expensive & Expansive Wi-Fi Routers & Cost effective \\
\hline Encryption & AES 128-Bits & $\begin{array}{c}\text { WPA2 (256-bit AES } \\
\text { Encryption) }\end{array}$ & Not Defined \\
\hline Security & Less Secure & $\begin{array}{l}\text { Highly Secure with } \\
\text { security standards }\end{array}$ & $\begin{array}{l}\text { Very high security and } \\
\text { protect from hackers }\end{array}$ \\
\hline IEEE Standard & IEEE 802.11 & IEEE $802.11 \mathrm{x}$ & Same as Wi-Fi \\
\hline
\end{tabular}

\section{Advantages of Li-Fi}

Li-Fi has bright future than Wi-Fi with its advanced technology policies. People are showing so much concern to know about Li-Fi. It has various advantages over Wi-Fi as follow:

- Super high Speed: The data speed of Li-Fi is 100 times faster than Wi-Fi and the internet browsing like uploading and downloading happens in few seconds [4].

- Wider Bandwidth: The Li-Fi has a wider bandwidth than Wi-Fi that is 1000 times more frequency. 
- High Security: It has high security than Wi-Fi as due to light, its signals can't penetrate through building walls like radio wave signals so it is very suitable to implement in high-security buildings like nuclear plants to prevent the hacking attacks on their private systems.

- Cost Effective: It is less expensive than Wi-Fi because its components like LED lamp and photodetector are available very easily at less rate than Wi-Fi router [3].

- Prevent Interference: It prevents interferences which occur due to overlapping of more than two access points of Wi-Fi.

- More efficient and reliable: It is more efficient and reliable network which can be easily recovered in case of failure of the system.

\section{Disadvantages of Li-Fi}

Although, Li-Fi replacing the Wi-Fi systems due to more list of advantages. But, it has some disadvantages also which are explained as follow:

- Its coverage area is very limited i.e. 10 meters only. But Wi-Fi coverage area is around 32 meters [2].

- Due to the interference of external sources like sunlight, its reliability may reduce.

- It works on Line-of -Sight mechanism and any disturbance in light can harm communication system and may lose the data.

- The communication medium of Li-Fi is light and any cut off in the light signal may interrupt the communication.

\section{Applications of Li-Fi}

$\mathrm{Li}$-Fi can be used in various fields to provide high-speed internet access and communication over the internet. Some future applications where Li-Fi can be used are explained as follow:

- It can be used in institutes, organizations to access high-speed internet browsing.

- It can be used in a high-security building like power plants, hospitals and in nuclear plants to protect their systems from any misuse by hackers [13].

- It can be used at traffic signals and implemented with traffic lights to know information about traffic and connected with the car's GPS system or any Smartphone device and reduce the chances of collision and accidents.

- It can be used in hill station areas where Wi-Fi implementation is very difficult to implement.

- It can be used in aircraft, underwater to provide communication links where communication is not possible without radar systems.

\section{Conclusion and Future Scope}

$\mathrm{Li}-\mathrm{Fi}$ is an optical wireless communication which is becoming a commercial need of end users as light is present everywhere and we can limit its wastage by integrating it with new technologies like Li-Fi to make over nation's future bright and brighter because of new and Nanotechnology based enhancements. Li-Fi is the new and interesting era for wireless communication using visual light signals. To make electronic communication fast and easy, Li-Fi is playing an important role and help to avoid cyber criminals to hack the internet access over the Li-Fi connection as happens in Wi-Fi connections. Network users are so much in love with internet surfing that they started hacking the private Wi-Fi connections for their own benefits. So, to provide high data security to wireless communication as well as to secure internet connections, Li-Fi is invented and also affordable by the internet users and very reliable. In coming years, it can be adopted by millions of 
organizations and to make daily life problems very easy as it can be accessed by any Smartphone device and will frequent the internet access to its users with high-speed data rate \& security as Li-Fi doesn't work outdoors so it is not for public due to sunlight interference effect and only used indoor and also has health and safety concerns as compared to Wi-Fi. Numerous of techniques and methods are launched and adopted to promote this novel wireless technology all over the world in future. Li-Fi Technology is just beyond the expectations and will lift the development of wireless networking and will help to track cyber criminals and provide resistance against internet intruders to protect confidential information from hackers and also it will avoid data manipulation attacks on transmitted data over the internet. To make network users free from data hacking phobia, Li-Fi will help to solve critical issues of today's networking world by avoiding electronic interference.

\section{Acknowledgements}

We would like to express our profound gratitude to all the Friends and Professors who support us a lot and also very grateful to all researchers for giving an idea in the formation of this paper on novel technology like Li-Fi. Hopefully, this paper will be a great initiative for the new researchers who want to do their research in this field.

\section{References}

[1] Jyoti Rani, Prerna Chauhan, Ritika Tripathi, "Li-Fi (Light Fidelity)-The future technology In Wireless communication", International Journal of Applied Engineering Research, Vol.7 No.11 (2012).

[2] Sinku U. Gupta, "Research on Li-Fi Technology\& Comparison of Li-Fi/Wi-Fi", International Journal of Scientific \& Engineering Research, Volume 4, Issue 12, December 2013.

[3] Neha S.Jaiswal, Payal S.Chopade, "Review of Li-Fi Technology: New Future Technology- Light Bulb to Access the Internet!", International Journal of Scientific \& Engineering Research, Volume 4, Issue 12, December-2013.

[4] Ravi Prakash, Prachi Agarwal, "The New Era of Transmission and Communication Technology: Li-Fi (Light Fidelity) LED \& TED Based Approach", International Journal of Advanced Research in Computer Engineering \& Technology (IJARCET) Volume 3, Issue 2, February 2014.

[5] Rahul R. Sharma, Raunak, and Akshay Sanganal, "Li-Fi Technology: Transmission of data through light", International Journal Computer Technology \& Applications (IJCTA), Volume 5 (1), pp: 150-154, February 2014.

[6] B.Narmada, P.Srinivasulu and P.Prasanna Murali Krishna, "High Efficient Li-Fi and Wi-Fi Technologies in Wireless Communication by Using Finch Protocol", International Journal of Engineering Trends and Technology (IJETT), Volume 12, Number 5, June 2014.

[7] Sumant Ku Mohapatra, Ramya Ranjan Choudhury, Pravanjan Das, "The Future Directions in Evolving WI-FI: Technologies", APPLICATIONS AND SERVICES", International Journal of Next-Generation Networks (IJNGN) Vol.6, No.3, September 2014.

[8] Atul M Gonsai, Nilesh N Soni, and NehaGoswami, "Emerging Wireless Technologies for Fast Data Transmission on Library Network", International Journal of Information Services and Technology, Vol.1, Issue No.1, December 2014, pp. 16-20.

[9] Harald Haas, "What is LiFi?", Journal of Lightwave Technology (IEEE), Volume 36, Issue 6, December 2015, pp: 1533 - 1544.

[10] Muhammad Usama, Kamran Saeed, Adnan Yousaf, “A Review on Nomadic Access of Li-Fi Technology”, Research Gate, March 2015.

[11] R.Karthika and S.Balakrishnan, "Wireless Communication using Li-Fi Technology", SSRG International Journal of Electronics and Communication Engineering (SSRG-IJECE) volume 2 Issue 3 March 2015. 
[12] Ala Al-Fuqaha, "Internet of Things: A Survey on Enabling Technologies, Protocols, and Applications", IEEE Communication Surveys \& Tutorials, Volume. 17, Issue. 4, 2015.

[13] Anurag Sarkar, Prof. Shalabh Agarwal, "Li-Fi Technology: Data Transmission through Visible Light", International Journal of Advance Research in Computer Science and Management Studies (IJARCSMS), Volume 3, Issue 6, June 2015.

[14] Shubham Chatterjee, Shalabh Agarwal, Asoke Nath, "Scope and Challenges in Light Fidelity (LiFi) Technology in Wireless Data Communication", International Journal of Innovative Research in Advanced Engineering (IJIRAE) , Issue 6, Volume 2, June 2015.

[15] http://newtecharticles.com/new-li-fi-technology-to-access-internet: accessed 30 May 2016.

[16] Bhawabhuti Mahavidyalaya Amgaon, Dist-Gondia, "An emerging technology of data transfer through light waves (Li-Fi)", International Journal of Recent Trends in Engineering \& Research (IJRTER), Volume 02, Issue 02; February- 2016.

[17] Mohammed Abdulmalek Ahmed, "Li-Fi: The Future Bright Technology in Wireless Communication", International Journal of Advanced Research in Computer Science and Software Engineering, Volume 6, Issue 3, March 2016.

[18] P.Loganathan, R.Dhilip Kumar, S.Ramachandran, "Light-Fidelity (Li-Fi) Technology: A Review", International Journal of Modern Trends in Engineering and Research (IJMTER), Volume 03, Issue 01, January 2016.

[19] Bhavya R., Lokesh M. R., “A Survey on Li-Fi Technology”, An International Journal of Engineering \& Technology, Vol. 3, No. 1, January 2016.

[20] Mrs. Gayatri .S, Dr.Arutchelvan G., " An Extensive comparison of Existing and Emerging Wireless Technology (WI-FI, WIMAX, GI-FI AND LI-FI)", International Research Journal of Engineering and Technology (IRJET), Volume 03, Issue 04 , April 2016.

[21] Prof. Amit K. Mishra, Mr. Lalit A. Pawar, Mr. Sandeep U. Gaikwad, Mr. Gaurav A. Sonawane, "Li-Fi: Wireless Communication Media",International Journal of Innovative Research in Electronics, Instruments and Control engineering, Vol. 4, Issue 2, February 2016.

[22] Mr. Shailendra Yadav, Mr. Pradeep Mishra, Miss. Minakshee Velapure,\& Prof.P.S.Togrikar, "LI-FI Technology for Data Transmission through LED", Imperial Journal of Interdisciplinary Research (IJIR) Vol-2, Issue-6, 2016.

[23] S. D. Fabiyi, "Li-Fi: A Full-Fledged Wireless Communication Technology", International Journal of Science and Research (IJSR), Volume 5 Issue 4, April 2016.

[24] Antriksh Borkar, "A Bidirectional Wireless CommunicationTechnology - Light Fidelity", International Journal of Innovative Research in Computer and Communication Engineering, Vol. 4, Issue 1, January 2016.

[25] M.Sowmiya, G.Visali, “A Study on LiFi Wireless Visible Light Communication”, International Journal of Engineering Development and Research (ijedr), Volume 4, Issue 1, 2016.

[26] J. Lidwina Jennifer, S. Jayanthy2 and J. Sujitha, "Li-Fi Technology based Fleet Vanguardand Security", Indian Journal of Science and Technology, Vol 9(11), March 2016.

[27] Gaurav Singh, Santoshkumar Yadav, Essakkimuthu Nadar, "Transmission of Data Using Li-Fi Technology", International Journal of Computer Science Trends and Technology (I JCST), Volume 4 Issue 2, Mar - Apr 2016.

[28] Sunita Saini, Dr.Yogesh Kumar Sharma, "LI-Fi the Most Recent Innovation in Wireless Communication", International Journal of Advanced research in Computer Science and Software Engineering, Volume 6, Issue 2, February 2016.

[29] http://www.wipro.com/blogs/light-fidelity-the-bright-future-of-5g-visible-light-communication-systems/Accessed on 14 August 2016.

[30] Gagandeep Kaur Virk, "Li-Fi: A New Communication Mechanism", International Journal of Computer Applications (IJCA), Volume 118, Issue 15, May 2015, pp: 1- 4. 
[31] http://www.techworld.com/big-data/what-is-li-fi-everything-you-need-know-3632764/- Accessed on 17 August 2016.

[32] M.A. Hadi, "Wireless Communication tends to Smart Technology Li-Fi and its comparison with Wi-Fi", American Journal of Engineering Research (AJER)", Volume 5, Issue 5, 2016, pp: 40-47.

[33] N.Navyatha, T.M.Prathyusha, V.Roja, M.Mounika, "Li-Fi (Light fidelity)-LED Based Alternative", International Journal of Scientific \& Engineering Research, Volume 4, Issue 5, May-2013, pp: 1039-1042.

[34] Mihir Chauhan, Aditya Kulai, "Li-Fi - Let There Be Light", International Journal of Engineering Trends and Technology (IJETT), Volume 28, Issue 4, October 2015, pp: 163-165.

[35] Prof. AmitK.Mishra, Mr. Lalit A. Pawar et.ol, “ Li-Fi: Wireless Communication Media”, International Journal of Innovative Electrical, Electronics, Instrumentation and Control Engineering, Volume 4, Issue 2, February 2016, pp:71-73.

[36] Izaz Ali Shah, Shahzeb Hayat, Ihtesham Khan, Imtiaz. Alam, Sadiq Ullah, Adeel Afridi, International Journal of Wireless and Microwave Technologies (IJWMT), "A Compact, Tri-Band and 9-Shape Reconfigurable Antenna for WiFi, WiMAX and WLAN Applications", PP.45-53, DOI: 10.5815/ijwmt.2016.05.05.

\section{Authors' Profiles}

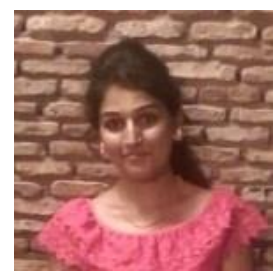

Ramandeep Kaur is a Research Scholar and completed her BTech from the college of Engg. \& Management, Kapurthala as well as MTech from CT group of institutions, Jalandhar. She has published various research papers in international as well as national journals like IEEE, IJCA and attends research conferences. Her interest of field is digital image processing \& Networking.

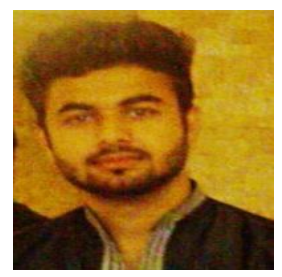

Himanshu Walia is a Research Scholar and completed his graduation (MTech) in computer science. He has published various research papers in international as well as national journals and attends various seminars and research conferences. His interest of field is Networking and working as software engineer in Simcomm Ltd.

How to cite this paper: Ramandeep Kaur, Himanshu Walia,"Review on Light Fidelity (Li-Fi) - An Advancement of Wireless Network", International Journal of Wireless and Microwave Technologies(IJWMT), Vol.7, No.3, pp.25-35, 2017.DOI: 10.5815/ijwmt.2017.03.03 